\title{
Monitoring the mammalian fauna of urban areas using remote cameras and citizen science
}

\author{
Victor Anton, ${ }^{*}$ Stephen Hartley, Andre Geldenhuis and Heiko U. Wittmer
}

\author{
School of Biological Sciences, Victoria University of Wellington, PO Box 600, Wellington 6140, New Zealand \\ ${ }^{*}$ Corresponding author. E-mail: victor.anton@vuw.ac.nz
}

Submitted: 27 August 2017; Received (in revised form): 15 January 2018; Accepted: 23 February 2018

\begin{abstract}
Remotely activated cameras are increasingly used worldwide to investigate the distribution, abundance and behaviour of animals. The number of studies using remote cameras in urban ecosystems, however, is low compared to use in other ecosystems. Currently, the time and effort required to classify images is the main constraint of this monitoring technique. To determine whether, or not, citizen science might help overcome this constraint, we investigated the engagement, accuracy and efficiency of citizen scientists providing crowd-sourced classifications of animal images recorded by remote cameras in Wellington, New Zealand. Classifications from individual citizen scientists were in $84.2 \%$ agreement with the classifications of professional ecologists. Aggregating the classifications from three citizen scientists per image, and excluding false triggers and unclassifiable classifications increased their overall accuracy to $97.6 \%$. Classifications by citizen scientists also improved if animal movement was highlighted in the images. The likelihood of citizen scientists correctly classifying images was influenced by their previous accuracy, their self-assessed confidence, and the species reported. Weighting the citizen scientist classifications based on their ability to correctly identify animals reduced from 3 to 2 the number of classifications required per sequence to classify $>95 \%$ of the photographs containing cats. Citizen science is an accurate and efficient approach for classifying remote camera data from urban areas, where most of the animals are familiar to the participants. We demonstrated how appropriate tools and accounting for the accuracy of citizen scientists, allows project managers to maximise the effort of citizen scientists while ensuring high-quality data.
\end{abstract}

Key words: Camera traps, animal identification, public engagement, conservation volunteers

\section{Introduction}

Remotely activated cameras are increasingly used worldwide to investigate behaviour, distribution and abundance of an array of animals (Meek et al. 2014). The availability, efficiency and user-friendly characteristics of remote cameras have contributed to calls for the establishment of a global network for monitoring biodiversity (Steenweg et al. 2017). The number of ecological studies using remote cameras in urban areas, however, is low compared to use in other ecosystems. For example, a literature search in June 2017 using the Web of Science Core Collection database for documents published between 2010 and 2016 containing the words 'Camera trap', 'Camera trap forest' and 'Camera trap urban' returned 2259, 713 and 45 publications, respectively.

The low number of urban studies using remote cameras is surprising given their potential to unravel critical ecological research questions. For example, remote cameras may assist urban ecologists to better understand the impact that invasive species have on native biodiversity, due to their ability to simultaneously monitor a wide range of animal species across multiple sites and over long periods of time (Elmqvist et al. 2013; Anton, Hartley, and Wittmer 2018). Ecologists are already using remote cameras under a wide range of conditions including harsh physical environments and locations with limited accessibility (Steenweg et al. 2017). The versatility of this monitoring

(c) The Author(s) 2018. Published by Oxford University Press.

This is an Open Access article distributed under the terms of the Creative Commons Attribution Non-Commercial License (http://creativecommons.org/ licenses/by-nc/4.0/), which permits non-commercial re-use, distribution, and reproduction in any medium, provided the original work is properly cited. For commercial re-use, please contact journals.permissions@oup.com 
technique facilitates cross-ecosystem studies that could elucidate how pets, human disturbances and other elements commonly found in urban environments influence community assemblies, predator-prey dynamics and animal behaviour (Faeth et al. 2005; Kikillus et al. 2017).

A potential constraint on the use of remote cameras, however, is the large number of images they may collect and the time and effort required to classify the species present in the images. Remote camera data often include poor quality images (e.g. blurry, pixelated, out of focus and over/underexposed pictures), images of animals camouflaged with the surrounding environment and partial views of animals (Meek et al. 2014; Swanson et al. 2016). Using appropriate camera deployment, settings and models can minimise the number of poor quality images (Nazir et al. 2017). False triggers, cameras triggered by objects other than target animals, is another challenge that researchers face while classifying remote camera data (Welbourne et al. 2016). False triggers can rapidly increase the number of images requiring classification to the point of unmanageable levels (Swinnen et al. 2014). Despite the recent improvement in algorithms trained to automatically identify false triggers or the animals recorded in the photographs (Norouzzadeh et al. 2017), human validation is still required for collating data from remote cameras (Kumar, Manohar, and Chethan 2015; He et al. 2016).

Citizen science, involving the general public in scientific research, is increasingly used for classifying large amounts of footage or photographs provided by remote sensing devices (Chandler et al. 2017). Data quality, a major concern in citizen science, can be maximised if citizen scientists are provided with the appropriate tools to accomplish their task (Ellwood, Crimmins, and Miller-Rushing 2017). For example, Horn et al. (2015) reported that the interface used for displaying photographs influenced the accuracy of citizen scientists to classifying common bird species found in North America. To ensure high-quality data, the performance of citizen scientists is often compared to that of professionals (Kosmala et al. 2016; McShea et al. 2016). If there are differences between the two groups, managers can apply an array of tools to achieve similar results to those provided by professionals (Lewandowski and Specht 2015; Kosmala et al. 2016).

In remote camera studies, the number of citizen scientists required to accurately classify the data is influenced by the goals and resources of the project (Swanson et al. 2016). For example, remote camera studies of rare or elusive species may require higher accuracy levels than studies looking at the community composition. Independent of project-specific characteristics, agreement among citizen scientists is considered a versatile tool for increasing the accuracy of citizen scientists (Swanson et al. 2016). The effort of citizen scientists, however, might be maximised by taking into account metadata associated with the individual citizen scientists and their past classifications (Kosmala et al. 2016). For example, participation experience (Kelling et al. 2015), expertise (Pocock et al. 2015), age (Delaney et al. 2008) and how confident citizen scientists are with their prediction (Crall et al. 2011) have previously been linked with the ability of citizen scientists to correctly identify animal species in similar projects. Due to the lower suite of mammalian species compared to urban ecosystems elsewhere (Baker and Harris 2007; Morgan, Waas, and Innes 2009; Widdows, Ramesh, and Downs 2015; Anton, Hartley, and Wittmer 2018), remote camera data collected in urban New Zealand are well suited to investigate algorithms for citizen scientists to reach the same levels of classification accuracy as professionals.

To determine whether, or not, citizen science may overcome the time and effort required to classify remote camera data from urban areas, we investigated the engagement, accuracy and efficiency of citizen scientists classifying remote camera data collected in Wellington, New Zealand.

\section{Methods \\ Data collection}

We used 4466 sequences of still photographs from remote cameras deployed at forested areas and private backyards in Wellington, New Zealand. Remote cameras (Bushnell 119537) were deployed for 3 months in 2014, as described by Anton, Hartley, and Wittmer (2018), and captured a sequence of three photographs per trigger over a 2-s period (henceforth a set of three images equals one sequence).

We uploaded all the sequences to a citizen science website (www.identifyanimals.co.nz) open to any member of the public. We promoted the website via local newspapers, social media and school visits. Upon arriving at the website, participants were required to create an account (username and password) before they could start classifying sequences. Participants also needed to report their age (years) and self-assess their knowledge of invasive mammals in New Zealand as poor, basic, good or excellent. Once registered, citizen scientists were able to classify the sequences into 1 of 15 categories available and report how confident they were with each classification (unsure, reasonable or very confident) (Fig. 1). Citizen scientists were also able to use a comment box on each sequence to classify the animal further (e.g. to the species level) or to report any concerns. The website included a tutorial page and a classification guide with 'how-to-use' instructions, but citizen scientists were not required to complete any training before starting to classify the sequences.

To estimate the accuracy and efficiency of citizen scientists, all sequences were classified by at least three citizen scientists, and by at least two different experts (i.e. professionals with formal qualifications in the field of ecology). If there was disagreement among expert classifications, the sequence was classified by Victor Anton.

For 1671 randomly selected sequences, we also provided a fourth image in which areas of change between the three original images were highlighted in purple in order to draw attention to potential animal movement in the sequence (Supplementary Appendix S1). This was achieved using the functions 'ImageChops.difference' and 'Image.blend', from the Python image library. We then compared the classification accuracy of users for sequences with and without the fourth image of highlighted movement.

\section{Data analysis}

We conducted all statistical analyses in $\mathrm{R}$ version 3.1.2 ( $\mathrm{R}$ Core Team 2016). To simplify the interpretation of the results, we excluded 2 of the 4466 sequences from the accuracy and efficiency analyses because, based on the comments provided by experts and citizen scientists, these sequences contained two different animals (i.e. hedgehogs and cats) and the user interface only allowed one option to be chosen. 


\section{Engagement of citizen scientists}

To study how the age and self-assessed ecological knowledge of the citizen scientists influenced engagement with the project, we fitted a generalised linear model. Engagement, the response variable, was measured as the total number of classifications provided by each citizen scientist. The explanatory variables were age and self-assessed ecological knowledge of the citizen scientist. We considered variables with $\mathrm{P}<0.05$ as significantly correlated with the number of classifications provided by each citizen scientist.

\section{Accuracy of citizen scientists}

We randomly selected three citizen scientist classifications per sequence and estimated the accuracy of individual citizen scientists and of their aggregated classifications. To estimate the accuracy of individual citizen scientists, we compared each classification to those provided by experts, and calculated the true positive rate (TPR or sensitivity) and positive predictive value (PPV) (Table 1). To estimate the accuracy of the aggregated classifications of citizen scientists, we identified the aggregated classification for each sequence based on the category with the highest number of citizen scientist classifications. If all three classifications were different, we randomly selected one of them as their aggregated classification. We considered a classification being 'correct' if the aggregated classification provided by citizen scientists agreed with the classification provided by experts.

We tested whether, or not, highlighting areas of movement across the 3 images of 1672 sequences influenced the ability of citizen scientists to make correct classifications. We randomly selected three citizen science classifications per sequence and per treatment (with and without the movement highlighted) and used Fisher's exact tests to evaluate statistical differences in the number of correct vs incorrect individual citizen scientist classifications between treatments.

We used generalised linear mixed models (GLMM) to investigate what metadata best predicts the likelihood of a citizen scientist making a correct classification. We ran the models using two-thirds of the sequences uploaded to the website $(n=2978)$. We used the complementary one-third of sequences to validate the efficiency of these models, as explained later. The response variable of the model was the accuracy of the classification provided by an individual citizen scientist (correct vs incorrect), for which we used the binomial family with log link. The explanatory variables of the model were age of the citizen scientists, their knowledge of New Zealand invasive mammals, the

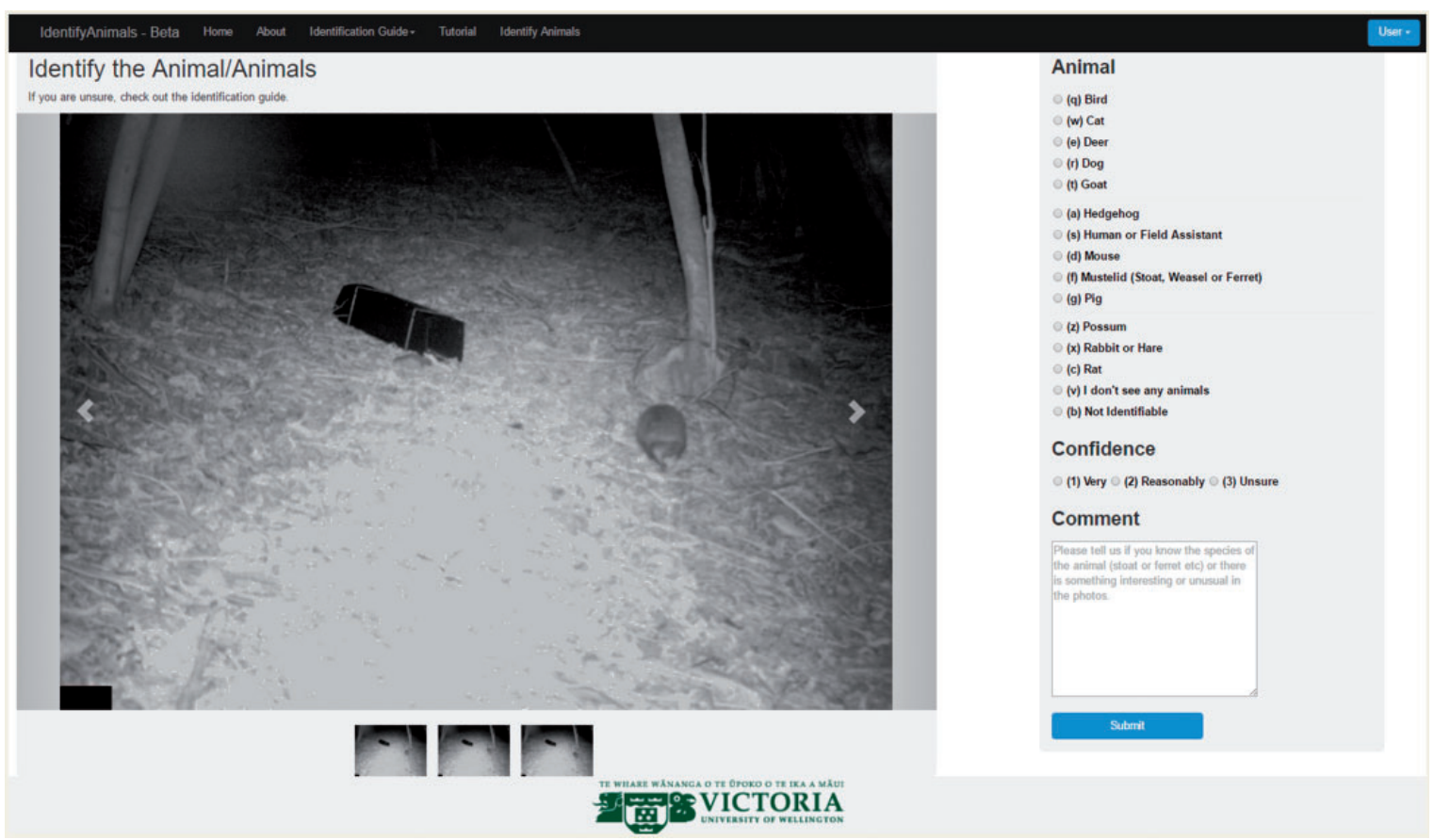

Figure 1: Snapshot of the interface of www.identifyanimals.co.nz used to classify the events. Citizen scientists could enlarge any of the three photographs (bottom of the screen) of each event. Citizen scientists needed to select one category and their confidence level (right-hand side of the screen) before submitting their classification. Citizen scientists were also able to classify the animal to the species level or report any anomaly using the comment box.

Table 1: Confusion matrix of the citizen scientist and expert classifications used to calculate the sensitivity and PPV of citizen scientists for category $i$ (e.g. $i=$ deer, pig, dog, etc.)

\begin{tabular}{llc}
\hline & $\begin{array}{c}\text { Expert classifications } \\
\text { of category } i\end{array}$ & $\begin{array}{c}\text { Expert classifications } \\
\text { different to category } i\end{array}$ \\
\hline Citizen scientist classifications of category $i$ & $\mathrm{a}$ & $\mathrm{b}$ \\
Citizen scientist classifications different to category $i$ & $\mathrm{c}$ & $\mathrm{d}$ \\
\hline
\end{tabular}

Sensitivity (or TPR) was calculated as: $a /(a+c)$. PPV was calculated as: $a /(a+b)$ 
confidence level they reported for the classification, previous accuracy (PPV) and number of classifications submitted by each citizen scientist. We estimated the previous accuracy of citizen scientists to classify each taxon category because the animals recorded in the photographs influence the ability of citizen

Table 2: Results of the GLM predicting number of classifications provided per citizen scientist based on their age and ecological knowledge (poor, basic, good or excellent)

\begin{tabular}{lccc}
\hline Variable & Estimate of $\beta$ & SE & P-value \\
\hline Intercept & 2.42 & 0.61 & $<0.001^{* * *}$ \\
Age & 0.03 & 0.01 & $<0.001^{* * *}$ \\
Ecological knowledge (basic) & 0.21 & 0.61 & 0.74 \\
Ecological knowledge (good) & 0.21 & 0.60 & 0.72 \\
Ecological knowledge (excellent) & 0.87 & 0.65 & 0.18 \\
\hline
\end{tabular}

The marginal $R^{2}$ of the model was 0.14 .

${ }^{* * *} \mathrm{P}<0.001$ scientists to correctly classify them (McShea et al. 2016). We calculated the previous accuracy of a citizen scientist per category for those categories (species) in which the citizen scientist had classified at least two previous sequences. For those citizen scientists that had not classified at least two sequences of that category, we used the average accuracy that all citizen scientists had in classifying that category (species). To improve numerical stability, we subtracted the mean of the variables age, previous accuracy and number of sequences classified per participant and divided them by their corresponding standard deviation (Gelman and Hill 2007). After running a Tukey's Honest Significant Difference, we simplified the variable ecological knowledge into 'poor or basic', 'good' and 'excellent' due to the lack of significant differences $(P>0.05)$ between citizen scientists with poor or basic ecological knowledge and their accuracy. We also used the category reported by the citizen scientists as a random effect because the ability of citizen scientists to correctly classify remote camera data is speciesspecific (McShea et al. 2016). We determined the best model to predict the likelihood of a citizen scientist being correct using
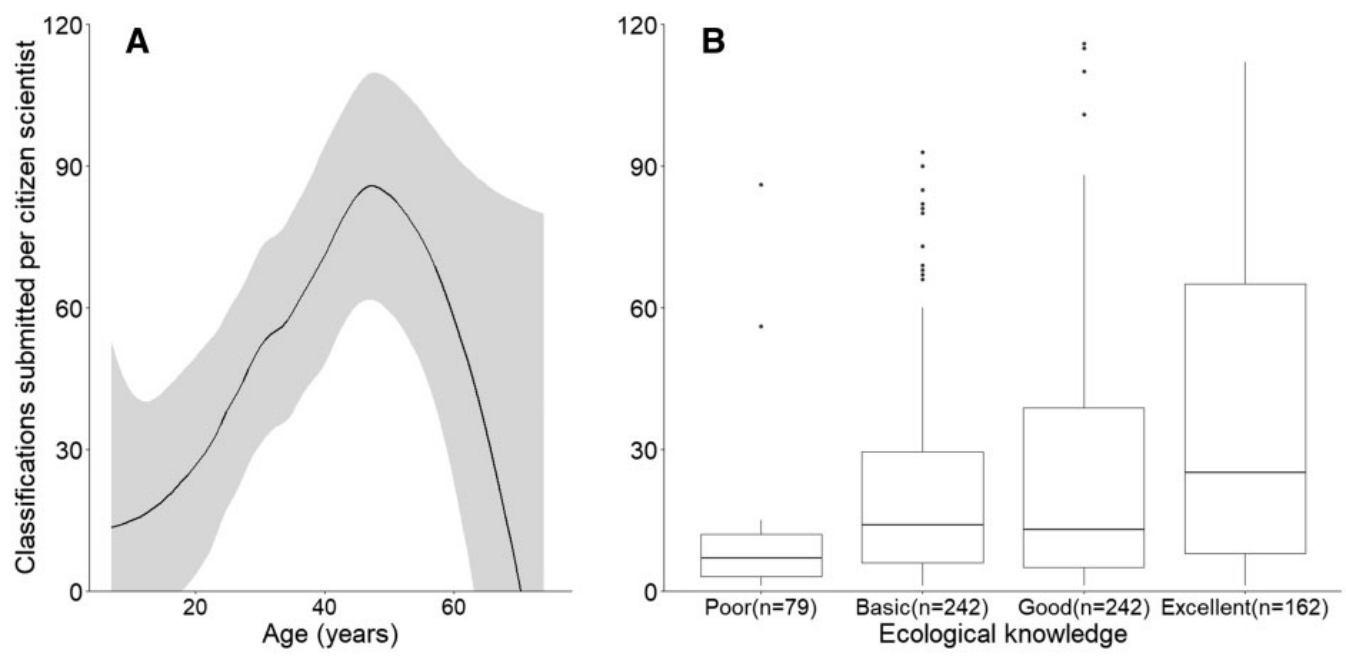

Figure 2: Engagement of citizen scientists according to (a) their age and (b) ecological knowledge. Engagement of citizen scientists is represented as the number of classifications submitted per citizen scientist. The loess method was used to estimate the mean number of classifications submitted per citizen scientist and the corresponding 95\% confidence intervals. Previous knowledge of New Zealand invasive mammals was used to represent the influence that ecological knowledge of citizen scientists had in the number of classifications submitted. Boxes in the boxplots represent the median and interquartile ranges.

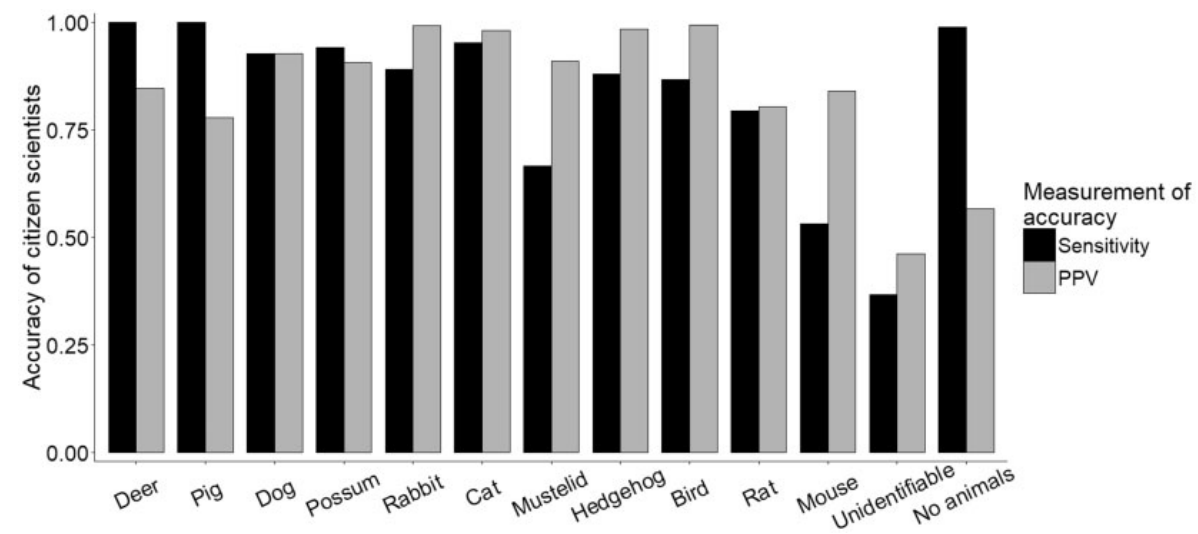

Figure 3: Performance of citizen scientists in classifying remote camera data collected from Wellington, New Zealand into 13 different categories. Performance was based on the comparison (sensitivity and PPV) between the aggregate classifications of three citizen scientists and at least two experts per event. Sensitivity is the proportion of events classified by experts as category ' $i$ ' that were correctly classified by citizen scientists, PPV is the proportion of events classified by citizen scientists as category ' $i$ ' that were correctly classified (see Table 1). Taxa are ordered according to average body size, from largest to smallest. 


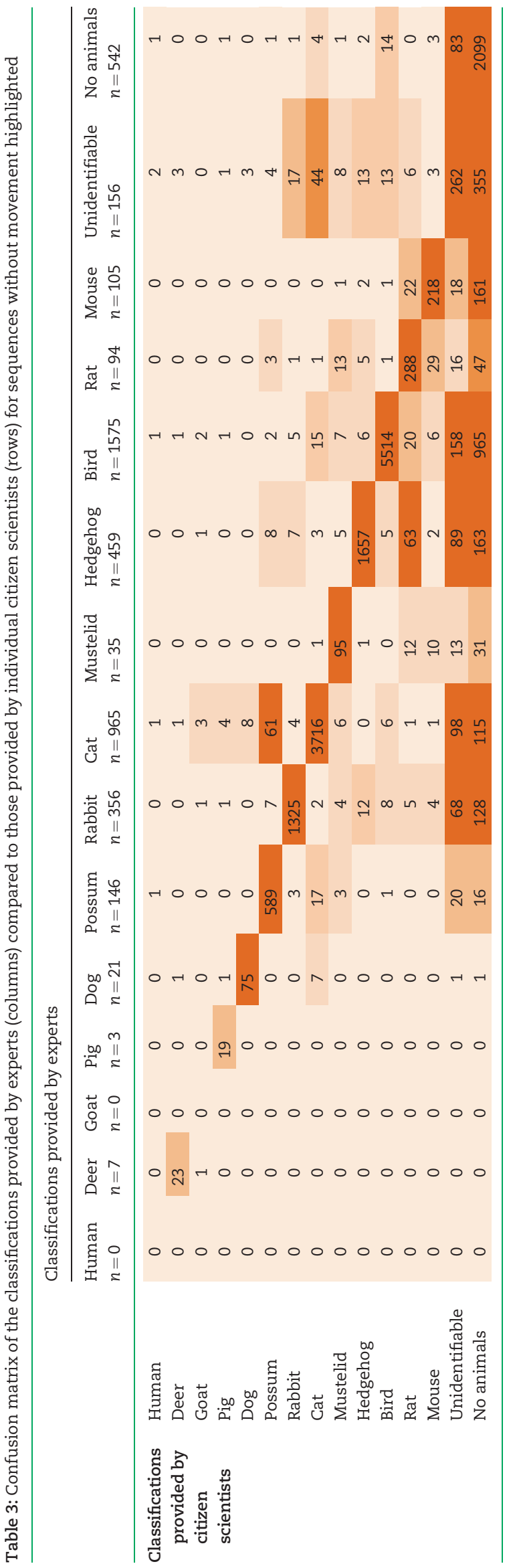

an information-theoretic approach to avoid over-fitting and to acknowledge uncertainty in model specification (Burnham and Anderson 2002). We generated all possible models $(n=16)$ and ranked them based on the Akaike's Information Criteria (AIC). We then averaged the models within four AIC of the 'best' model, using the MULMN package, to determine a parsimonious meta-model with the best overall support from the data (Arnold 2010; Barton 2015). We determined the influence of each variable for predicting the likelihood of a citizen scientist being correct based on the parameter estimates and standard errors of the averaged model. We used the marginal and conditional $R^{2}$ (Nakagawa and Schielzeth 2013) to estimate goodness of fit of the meta-model.

\section{Efficiency of citizen scientists}

We determined whether weighting the classifications, based on metadata associated with the accuracy of citizen scientists, influenced the number of citizen scientists required to correctly classify sequences containing invasive mammals commonly found in urban areas of New Zealand. Following Morgan, Waas, and Innes (2009), we considered the following species as common mammalian species of New Zealand urban areas: possums (Trichosurus vulpecula), cats (Felis catus), mustelids (Mustela spp.), hedgehogs (Erinaceus europaeus), rats (Rattus spp.) and mice (Mus musculus). Based on expert classifications we selected one-third of the sequences containing these invasive species to validate whether weighting the classifications had an effect on the sensitivity (TPR) of citizen scientists' classifications.

We weighted the classifications provided by citizen scientists based on the likelihood that each citizen scientists had of being correct, as predicted by the averaged GLMM, using the predict function from the 'Ime4' package (Bates et al. 2015). We, then, calculated the most likely classification based on the predicted accuracy of each citizen scientist. For example, if three citizen scientists provided classifications ('cat', 'cat' and 'possum') with modelled individual accuracies of $60 \%, 60 \%$ and $90 \%$, respectively, possum was selected as the aggregated classification because the likelihood of getting this set of classifications if the image contained a possum $(0.4 \times 0.4 \times 0.9)$ was greater than the likelihood of getting these classifications if the image contained a cat $(0.6 \times 0.6 \times 0.1)$. We used Fisher's exact tests to compare the proportion of sequences correctly classified by citizen scientists based on two and three weighted and unweighted classifications.

\section{Results}

\section{Engagement of citizen scientists}

A total of 504 citizen scientists submitted 24956 classifications over the 7 months that our website was active. The classifications submitted by the top 10 contributors represented $34.5 \%$ of the total classifications received. The number of classifications that citizen scientists submitted was significantly correlated with their age $(P<0.001)$ but not with their self-assessed knowledge of invasive mammals (Table 2). Citizen scientists between 35 and 55 years old provided more classifications than younger or older volunteers (Fig. 2).

\section{Accuracy of citizen scientists}

Classifications from individual citizen scientists were in $84.2 \%$ agreement with the classifications provided by experts. The accuracy of individual citizen scientists was influenced by the 


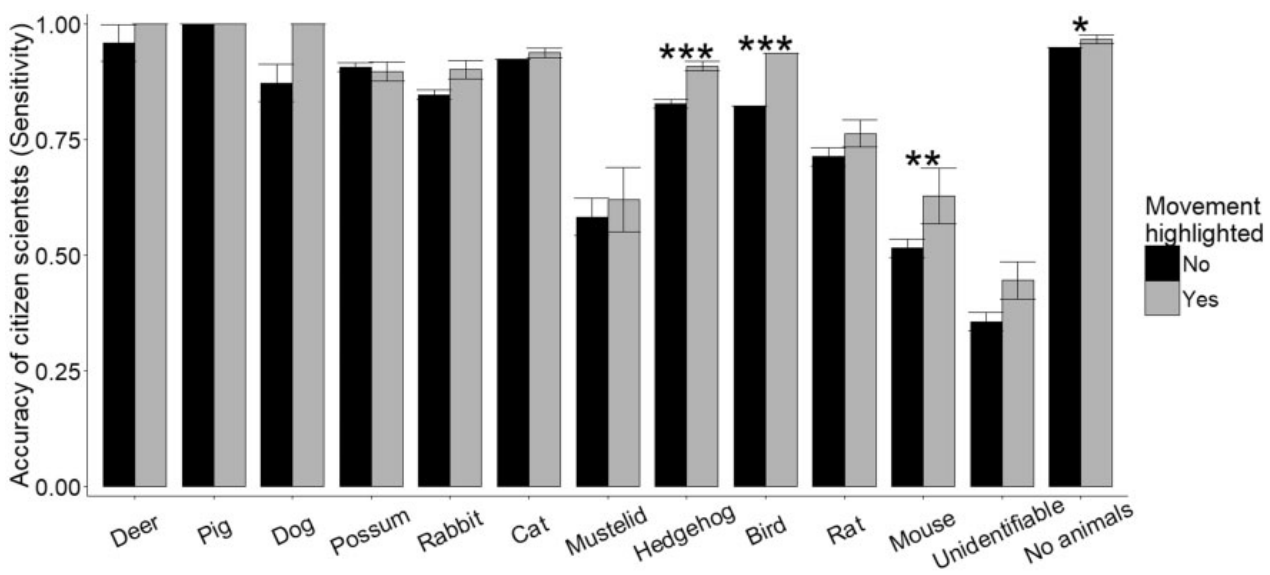

Figure 4: Comparison between the accuracy of citizen scientists to correctly classify events, with and without the movement highlighted, into 13 categories. Accuracy (sensitivity) was based on the comparison between the aggregated classifications of three citizen scientists and at least two experts per event. The events were collected using remote cameras from Wellington, New Zealand. Error bars represent standard errors; significant differences in the accuracy between events with and without movement highlighted are represented as ${ }^{*},{ }^{* *}$ and ${ }^{* * *}(P<0.05,<0.01$ and $<0.001$, respectively).

animal recorded in the photographs (Fig. 3); for example, citizen scientists correctly classified $>90 \%$ of the sequences containing deer or cats but $<70 \%$ of the sequences containing mustelids or mice. Citizen scientists often misclassified, as false triggers, sequences that experts were unable to classify and those containing birds (48.4\% and $14.4 \%$, respectively) (Table 3). Excluding the false triggers and unclassifiable classifications, individual citizen scientists were in $97.0 \%$ agreement with the classifications provided by experts.

The aggregated classification of citizen scientist increased the number of images correctly classified. For example, the aggregated classification of three citizen scientists was in $87.8 \%$ agreement with the classification of experts, $3.6 \%$ more than the classifications of individual citizen scientists. Excluding false triggers and unclassifiable sequences, the aggregated classifications of citizen scientists were in $97.6 \%$ agreement with the classification of experts.

The number of citizen scientists that used the comment box to classify the animal to species level was influenced by the animal recorded in the photograph. The proportion of citizen scientists that classified lagomorphs, mustelids, birds and rats to the species level were $9.4 \%, 18.7 \%, 17.6 \%$ and $3.7 \%$, respectively (Supplementary Appendix S2).

Highlighting areas of movement in the photographs had a positive effect on the ability of citizen scientists to correctly classify sequences. The sensitivity of the aggregated classification of three citizen scientists was, on average, $7.8 \pm 0.6 \%$ greater for those sequences with the movement highlighted compared to sequences without the movement highlighted. In particular, highlighting areas of movement significantly improved identification of hedgehogs, birds, mice and false triggers (Fig. 4).

The classification accuracy of individual citizen scientists may be predicted by their self-declared confidence with the classification, and their previous accuracy. Based on the metamodel used to predict the likelihood of being correct, citizen scientists that were 'reasonably confident' and 'very confident' with their classification were $12.9 \pm 1.8 \%$ and $36.9 \pm 1.5 \%$ more likely $(P<0.05)$ of being correct compared to those unsure with their classifications (Table 4). Citizen scientists were also more likely to correctly classify a sequence $(P<0.05)$ if they had been accurate in classifying other sequences of the same category previously. Previous accuracy and the
Table 4: Output of the meta-model used to predict the likelihood that citizen scientists had of correctly classifying a sequence (marginal $R^{2}=0.14$ and conditional $R^{2}=0.42$ )

\begin{tabular}{lccc}
\hline Model term & Estimate of $\beta$ & SE & P-value \\
\hline Intercept & 0.33 & 0.35 & 0.35 \\
Age (years) & 0.00 & 0.02 & 0.97 \\
Ecological knowledge (good) & 0.07 & 0.08 & 0.38 \\
Ecological knowledge (excellent) & 0.12 & 0.11 & 0.30 \\
Confidence level (reasonable) & 1.35 & 0.11 & $<0.001^{* * *}$ \\
Confidence level (very confident) & 2.09 & 0.12 & $<0.001^{* * *}$ \\
Previous accuracy (\%) & 0.50 & 0.05 & $<0.001^{* * *}$ \\
Number of classifications & 0.00 & 0.02 & 0.88 \\
\hline
\end{tabular}

The most parsimonious models were estimated by averaging the models with the lowest AIC ( $\triangle 4$ AIC). The fixed effects of the model were: age, confidence level (unsure, reasonable or very confident), ecological knowledge (poor \& basic, good or excellent) and previous accuracy of the citizen scientists. The category reported by the citizen scientist (e.g. cat, rat or mouse) was treated as a random effect.

${ }^{* * *} \mathrm{P}<0.001$.

likelihood of a citizen scientist being correct followed a linear relationship (Fig. 5).

\section{Efficiency of citizen scientists}

The number of citizen scientists required to correctly classify remote camera data was influenced by the desired levels of accuracy and by the species of interest. For example, one citizen scientist per sequence was sufficient to correctly classify $>90 \%$ of the sequences containing images of possums or cats (Table 5).

Weighting the citizen scientist classifications based on their previous accuracy and self-assessed confidence levels did not statistically increase the accuracy of classifying common invasive mammals of New Zealand urban areas. However, the classification accuracy for these species never decreased after weighting classifications. Furthermore, depending of the desired accuracy levels, weighting the classifications reduced the number of citizen scientists required per sequence. For example, weighting the citizen scientist classifications reduced from 3 to 2 the number of classifications required per sequence to classify $>95 \%$ of the photographs containing cats. 

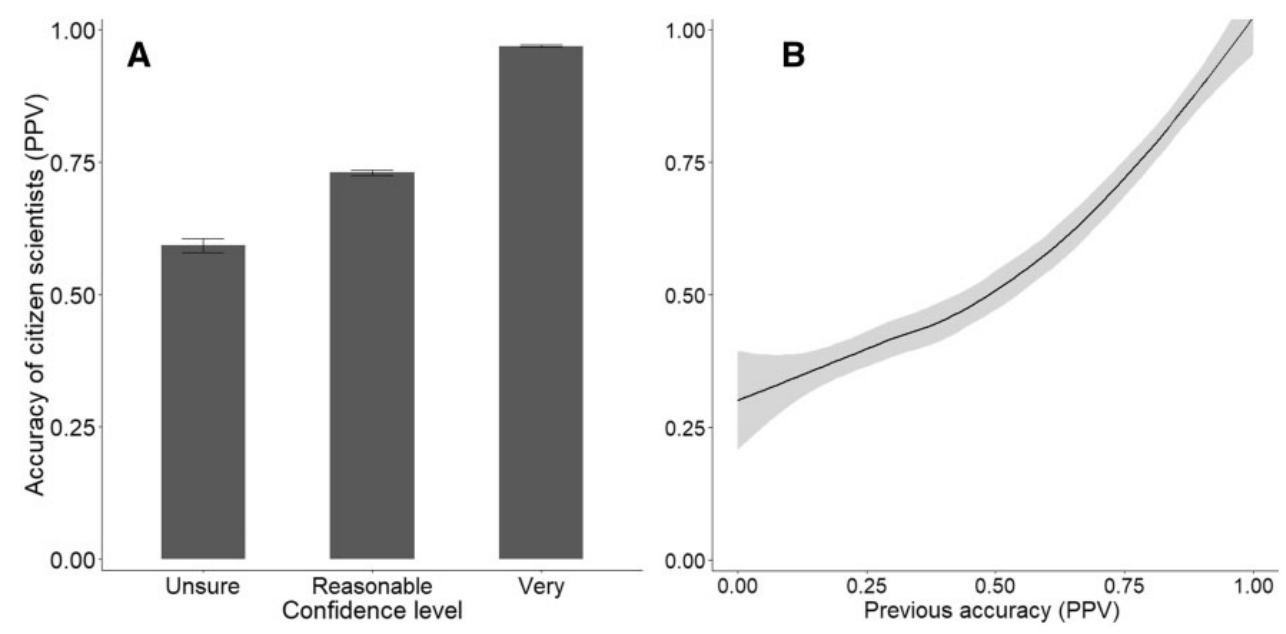

Figure 5: Accuracy of citizen scientists classifying remote camera data based on (a) the level of confidence they reported for each classifications and (b) their previous accuracy. Accuracy (PPV) was based on the proportion of events in which the citizen scientist classification matched the classification of experts. Mean accuracy and standard errors are represented for different levels of confidence citizen scientists had with their classification. The loess method was used to estimate the PPV of citizen scientists in relation to their previous accuracy.

Table 5: Number of sequences containing common mammalian introduced predators of New Zealand and the proportion of sequences correctly classified by one, two and three citizen scientists based on weighted and unweighted classifications

\begin{tabular}{lcccccc}
\hline Animal & Sequences & $\begin{array}{c}\text { One citizen } \\
\text { scientist (\%) }\end{array}$ & $\begin{array}{l}\text { Two citizen } \\
\text { scientists (\%) }\end{array}$ & $\begin{array}{l}\text { Two citizen scientists } \\
\text { with model weighting (\%) }\end{array}$ & $\begin{array}{l}\text { Three citizen } \\
\text { scientists (\%) }\end{array}$ & $\begin{array}{l}\text { Three citizen scientists } \\
\text { with model weighting (\%) }\end{array}$ \\
\hline Possum & 49 & 93.9 & 91.8 & 91.8 & 93.9 & 93.9 \\
Cat & 322 & 92.2 & 94.1 & 96.6 & 96.8 & 97.8 \\
Mustelid & 12 & 75.0 & 66.6 & 75.0 & 75.0 & 75.0 \\
Hedgehog & 153 & 83.7 & 85.0 & 88.9 & 88.9 & 91.5 \\
Rat & 32 & 75.0 & 81.3 & 61.3 & 84.4 & 87.5 \\
Mouse & 35 & 54.3 & 57.1 & 68.6 & 54.3 & 77.1 \\
\hline
\end{tabular}

Classifications were weighted based on the animal citizen scientists reported, their level of confidence and their previous accuracy. Proportions in bold indicate an increase in the accuracy of citizen scientists after weighting their classifications.

\section{Discussion and conclusion \\ Engagement of citizen scientists}

Citizen science is an important tool to increase nature awareness in urban environments (Wei, Lee, and Wen 2016). Online citizen science projects provide an easy-to-access opportunity to engage large segments of the general public in scientific research (Kosmala et al. 2016). Involving large numbers of people in citizen science projects thus requires understanding their motivations for participation as well as level of engagement (Singh et al. 2014). In our study, citizen scientists differed widely in their level of engagement; most volunteers classified less than 10 sequences while a few individuals $(n=3)$ classified over 1000 sequences. These results are consistent with previous studies showing that a small number of citizen scientists often provide the majority of contributions (Laut et al. 2017). Understanding demographic attributes of the most engaged citizen scientists may help improve and homogenise project participation (Lewandowski and Specht 2015; Laut et al. 2017).

In our study, professionals aged 35-55 years old were most engaged with the project website. Age of these highly engaged citizen scientists was lower than the average age of participants commonly involved in conservation and restoration activities (e.g. planting, weeding or wildlife monitoring) (Peters, Hamilton, and Eames 2015). This may suggest that modern technologies can be used effectively to engage with demographic groups that are usually underrepresented in conservation activities.

\section{Accuracy of citizen scientists}

The aggregated classifications from three citizen scientists were in $87.8 \%$ agreement with the classification of experts. This accuracy was greater than that reported in similar projects (e.g. Kosmala et al. 2016, McShea et al. 2016). The relatively low number of categories in our project may explain the increased accuracy. Specifically, we enabled citizen scientists to classify sequences into 15 different categories, substantially less than the 48 and 22 categories to choose from in remote camera studies by Swanson et al. (2016) and McShea et al. (2016), respectively. While this meant that some animals in our study were not classified at the species level, the ecological benefits of more detailed classifications were likely low compared to benefits of not overwhelming citizen scientists with demands for knowledge usually limited to experts which could risk reducing their engagement.

The high overall accuracy found in our study may also have been influenced by the familiarity citizen scientists had with 
the animals recorded. The fauna found in urban environments in New Zealand is relatively species-poor, particularly in mammals (Morgan, Waas, and Innes 2009; Anton, Hartley, and Wittmer 2018). However, a limited number of 'urban-adaptable' species have become widespread and locally abundant in cities all over the world, a common phenomenon known as biotic homogenisation (McKinney 2006). Our results thus highlight that involving citizen scientists in remote camera studies may be particularly suitable for urban environments because the general public is often familiar with most of the animals found in urban areas.

\section{Efficiency of citizen scientists}

Studies based on remote camera data should try to engage as many citizen scientists as possible because increased participation is linked to improved accuracy classifying images (Swanson et al. 2016). To make best use of volunteer efforts, project managers need to define the minimum number of citizen scientists required to achieve desired accuracy levels. Consistent with McShea et al. (2016) and Swanson et al. (2016), our results highlight that the number of citizen scientists required per sequence is category specific. We also showed how self-assessed confidence and previous accuracy can be used to reduce the number of classifications required without compromising data quality (Table 5). Weighting classifications of citizen scientists based on their ability to correctly identify animals may be particularly beneficial to increase volunteer efficiency for studies of complex ecological systems or species that are often misclassified by the general public (He et al. 2016). However, experts may be required to classify categories that, independent of the number of citizen scientist classifications received, do not reach desired levels of accuracy.

Remote cameras are a relatively novel technology for monitoring wildlife in urban environments. Remote cameras have great potential to further our understanding of topical research including invasive species, human-wildlife conflicts (Kahle, Flannery, and Dumbacher 2016; Murray et al. 2016), animal behaviour (Widdows, Ramesh, and Downs 2015) and trophic dynamics (Inger et al. 2016) in cities. Improvements in the capabilities of the cameras (Hobbs and Brehme 2017) and gradual decrease in equipment costs (Nazir et al. 2017) will continue to facilitate the use of remote cameras to better understand relationships between fauna, people and the urban landscape. We highlighted how citizen scientists can efficiently classify vast amounts of data, the main constrain of remote camera technology. However, vandalism, privacy issues, study design and camera specifications and deployment should be taken into consideration before using remote cameras in urban environments (Anton, Hartley, and Wittmer 2018; O'Connor et al. 2017).

In conclusion, involving citizen scientists with the use of remote cameras may facilitate a wider use of this technology including in urban areas while simultaneously improving knowledge of participants about wildlife (e.g. Forrester et al. 2017). To maximise the efficiency of volunteer' efforts while ensuring high-quality data, project managers need to account for the accuracy of citizen scientists and provide them with appropriate tools that facilitate data classification. Used in this way, citizen scientists can provide invaluable help classifying the large amount of data collected by camera studies.

\section{Supplementary data}

Supplementary data are available at JUECOL online.

\section{Acknowledgements}

We thank all 504 citizen scientists for their help classifying our camera data and Jason Preble, Johannes Fischer and Amy Brasch for comments on an earlier version of this manuscript. Victor Anton is grateful for a tuition scholarship from Victoria University of Wellington. We appreciate comments from two anonymous reviewers that greatly improved our manuscript.

\section{Funding}

Funding for this project was provided by Victoria University of Wellington, the Centre for Biodiversity and Restoration Ecology (CBRE) and Wellington City Council.

Conflict of interest statement. None declared.

\section{References}

Anton, V., Hartley, S., and Wittmer, H. (2018) 'Evaluation of Remote Cameras for Monitoring Multiple Invasive Mammals in New Zealand', New Zealand Journal of Ecology, 42: 1-6.

Arnold, T. W. (2010) 'Uninformative Parameters and Model Selection Using Akaike's Information Criterion', The Journal of Wildlife Management, 74: 1175-8.

Baker, P. J., and Harris, S. (2007) 'Urban Mammals: What Does the Future Hold? An Analysis of the Factors Affecting Patterns of Use of Residential Gardens in Great Britain', Mammal Review, 37: 297-315.

Barton, K (2015) MuMIn: Multi-Model Inference. R Package, Version 1.15.6. $<$ https://CRAN.R-project.org/package=MuMIn $>$ accessed 21 November 2017.

Bates, D. et al. (2015) 'Fitting Linear Mixed-Effects Models Using lme4', Journal of Statistical Software, 67: 1-48.

Burnham, K. P., and Anderson, D. R. (2002) Model Selection and Multimodel Inference: A Practical Information-Theoretic Approach. Springer Science \& Business Media.

Chandler, M. et al. (2017) 'Involving Citizen Scientists in Biodiversity Observation', The GEO Handbook on Biodiversity Observation Networks, pp. 211-237. Springer International Publishing.

Crall, A. W. et al. (2011) 'Assessing Citizen Science Data Quality: An Invasive Species Case Study', Conservation Letters, 4: 433-42.

Delaney, D. G. et al. (2008) 'Marine Invasive Species: Validation of Citizen Science and Implications for National Monitoring Networks', Biological Invasions, 10: 117-28.

Ellwood, E. R., Crimmins, T. M., and Miller-Rushing, A. J. (2017) 'Citizen Science and Conservation: Recommendations for a Rapidly Moving Field', Biological Conservation, 208: 1-4.

Elmqvist, T. et al. (2013) Urbanization, Biodiversity and Ecosystem Services: Challenges and Opportunities. Springer.

Faeth, S. H. et al. (2005) 'Trophic Dynamics in Urban Communities', BioScience, 55: 399.

Forrester, T. D. et al. (2017) 'Creating Advocates for Mammal Conservation through Citizen Science', Biological Conservation, 208: 98-105.

Gelman, A., and Hill, J. (2007) Data Analysis Using Regression and Multilevel/Hierarchical Models. Cambridge University Press.

$\mathrm{He}$, Z. et al. (2016) 'Visual Informatics Tools for Supporting Large-Scale Collaborative Wildlife Monitoring with Citizen Scientists', IEEE Circuits and Systems Magazine, 16: 73-86. 
Hobbs, M. T., and Brehme, C. S. (2017) 'An Improved Camera Trap for Amphibians, Reptiles, Small Mammals, and Large Invertebrates', PLoS One, 12: e0185026.

Horn, G. et al. (2015) 'Building a Bird Recognition App and Large Scale Dataset with Citizen Scientists: The Fine Print in Fine-Grained Dataset Collection', Proceedings of the IEEE Conference on Computer Vision and Pattern Recognition, 595-604.

Inger, R. et al. (2016) 'Ecological Role of Vertebrate Scavengers in Urban Ecosystems in the UK', Ecology and Evolution, 6: 7015-23.

Kahle, L. Q., Flannery, M. E., and Dumbacher, J. P. (2016) 'Bird-Window Collisions at a West-Coast Urban Park Museum: Analyses of Bird Biology and Window Attributes from Golden Gate Park, San Francisco', PLoS One, 11: e0144600.

Kelling, S. et al. (2015) 'Can Observation Skills of Citizen Scientists Be Estimated Using Species Accumulation Curves?', PLoS One, 10: e0139600.

Kikillus, K. H. et al. (2017) 'Research Challenges and Conservation Implications for Urban Cat Management in New Zealand', Pacific Conservation Biology, 23: 15-24.

Kosmala, M. et al. (2016) 'Assessing Data Quality in Citizen Science', Frontiers in Ecology and the Environment, 14: 551-60.

Kumar, Y. H. S., Manohar, N., and Chethan, H. K. (2015) 'Animal Classification System: A Block Based Approach', Procedia Computer Science, 45: 336-43.

Laut, J. et al. (2017) 'Increasing Citizen Science Contribution Using a Virtual Peer', Journal of the Association for Information Science and Technology, 68: 583-93.

Lewandowski, E., and Specht, H. (2015) 'Influence of Volunteer and Project Characteristics on Data Quality of Biological Surveys', Conservation Biology, 29: 713-23.

McKinney, M. L. (2006) 'Urbanization as a Major Cause of Biotic Homogenization', Biological Conservation, 127: 247-60.

McShea, W. J. et al. (2016) 'Volunteer-Run Cameras as Distributed Sensors for Macrosystem Mammal Research', Landscape Ecology, 31: 55-66.

Meek, P. et al. (2014) Camera Trapping: Wildlife Management and Research. Csiro Publishing.

Morgan, D. K., Waas, J. R., and Innes, J. G. (2009) 'An Inventory of Mammalian Pests in a New Zealand City', New Zealand Journal of Zoology, 36: 23-33.

Murray, M. H. et al. (2016) 'Urban Compost Attracts Coyotes, Contains Toxins, and May Promote Disease in Urban-Adapted Wildlife', EcoHealth, 13: 285-92.

Nakagawa, S., and Schielzeth, H. (2013) 'A General and Simple Method for Obtaining $R \quad 2$ from Generalized Linear
Mixed-Effects Models', Methods in Ecology and Evolution, 4: 133-42.

Nazir, S. et al. (2017) 'WiseEye: Next Generation Expandable and Programmable Camera Trap Platform for Wildlife Research', PLoS One, 12: e0169758.

Norouzzadeh, M. S. et al. (2017) 'Automatically Identifying Wild Animals in Camera Trap Images with Deep Learning', arXiv, 1703.05830.

O'Connor, K. M. et al. (2017) 'Camera Trap Arrays Improve Detection Probability of Wildlife: Investigating Study Design Considerations Using an Empirical Dataset', PLoS One, 12: e0175684.

Peters, M. A., Hamilton, D., and Eames, C. (2015) 'Action on the Ground: A Review of Community Environmental Groups' Restoration Objectives, Activities and Partnerships in New Zealand', New Zealand Journal of Ecology, 39: 179-89.

Pocock, M. J. O. et al. (2015) 'The Biological Records Centre: A Pioneer of Citizen Science', Biological Journal of the Linnean Society, 115: 475-93.

R Core Team. (2016) R: A Language and Environment for Statistical Computing. R Foundation for Statistical Computing. Version 3.1.2. Vienna, Austria. www.R-project.org/.

Singh, N. J. et al. (2014) 'Tackling the Motivation to Monitor: success and Sustainability of a Participatory Monitoring Program', Ecology and Society, 19: 7.

Steenweg, R. et al. (2017) 'Scaling up Camera Traps: Monitoring the Planet's Biodiversity with Networks of Remote Sensors', Frontiers in Ecology and the Environment, 15: 26-34.

Swanson, A. et al. (2016) 'A Generalized Approach for Producing, Quantifying, and Validating Citizen Science Data from Wildlife Images', Conservation Biology, 30: 520-31.

Swinnen, K. R. R. et al. (2014) 'A Novel Method to Reduce Time Investment When Processing Videos from Camera Trap Studies', PLoS One, 9: e98881.

Wei, J. W., Lee, B. P. Y., and Wen, L. B. (2016) 'Citizen Science and the Urban Ecology of Birds and Butterflies - A Systematic Review', PLoS One, 11: e0156425.

Welbourne, D. J. et al. (2016) 'How Do Passive Infrared Triggered Camera Traps Operate and Why Does It Matter? Breaking down Common Misconceptions', Remote Sensing in Ecology and Conservation, 2: 77-83.

Widdows, C. D., Ramesh, T., and Downs, C. T. (2015) 'Factors Affecting the Distribution of Large Spotted Genets (Genetta tigrina) in an Urban Environment in South Africa', Urban Ecosystems, 18: 1401-13. 